\title{
Introduction to the Special Issue on Highlights of ACM Intelligent User Interface (IUI) 2019
}

This special issue presents the extended version of seven papers from the ACM Intelligent User Interface (IUI) 2019 international conference that was held in Los Angeles, California, from March 16 to 20 , 2019. ACM IUI 2019 was the 24 th IUI conference, continuing its tradition of being the main international forum for reporting outstanding research at the intersection of human-computer interaction and artificial intelligence.

Along with a record number of delegates attending, the IUI 2019 conference received a record number of 282 full and short paper submissions from all over the world, of which 71 were accepted by the international program committee (acceptance rate of $25 \%$ ).

The final conference program included 55 long papers and 16 short papers, 43 posters, 19 demos, and 12 student consortium papers. In addition, building on the strong tradition of collaboration between IUI and the ACM TiiS journal, 9 TiiS papers published by the journal in 2018 were also presented at the conference.

The program committee continued the tradition by inviting authors of selected papers, considered among the best papers accepted into the program of ACM IUI 2019, to submit to this special issue. The authors of selected papers were invited to extend their work with at least $30 \%$ new material for consideration. The extended versions of the papers have gone through a new review process by at least three reviewers, often with multiple rounds of revision.

These extended versions allow the authors to elaborate on their excellent lines of research relevant to the TiiS community. As a result of this review process, we are excited to introduce the selected seven papers to the community.

ACM IUI 2019 had a focus on explainable artificial intelligence and interactive machine learning, which is also an important area covered by TiiS. Therefore, it is not surprising that our special issue includes several articles that touch upon this topic.

In "A Method and Analysis to Elicit User-Reported Problems in Intelligent Everyday Applications," the authors aim to investigate and assess problems reported by users in intelligent everyday applications. They analyze user reviews of four well-known apps that can be attributed to the apps' algorithmic decision making, then enrich this data with users' coping and support strategies through a follow-up online survey. However, they found problems and strategies related to content, algorithm, user choice, and feedback.

"Progressive Disclosure: When, Why, and How Do Users Want Algorithmic Transparency Information?" takes an empirical user-centric approach to investigate how to design transparent

\section{ACM Reference format:}

Oliver Brdiczka, Duen Horng Chau, Minsuk Kahng, and Gaëlle Calvary. 2020. Introduction to the Special Issue on Highlights of ACM Intelligent User Interface (IUI) 2019. ACM Trans. Interact. Intell. Syst. 10, 4, Article 27 (November 2020), 2 pages.

https://doi.org/10.1145/3429946

The reviewing of this article was managed by special issue associate editors Oliver Brdiczka, Duen Horng Chau, Minsuk Kahng, Gaëlle Calvary.

(C) 2020 Copyright held by the owner/author(s).

2160-6455/2020/11-ART27

https://doi.org/10.1145/3429946 
systems. Through four studies of an emotional analytics system, they assess user reactions to transparency feedback. Based on the study results, they developed new user-centric progressive disclosure principles for presenting transparency in intelligent systems.

The following two articles particularly focus on explanations in recommender systems, which has been one of the popular topics at IUI.

"Algorithmic and HCI Aspects for Explaining Recommendations of Artistic Images" studies the effects of explaining recommender systems for artistic images by conducting two user studies on Amazon Mechanical Turk. The authors evaluate different levels of explainability, combined with different algorithms, finding that explanations of recommendations in the image domain can increase user satisfaction, perception of explainability, and relevance.

In "Generating and Understanding Personalized Explanations in Hybrid Recommender Systems," the authors present a personalized hybrid recommender engine that integrates multiple sources of information and generates explanations with a variety of styles. They conduct a crowdsourced user study to evaluate the effects of explanation style, number, and format, as well as personality characteristics on user preferences for explanations.

IUI always includes amazing real-world applications of intelligent user interfaces. The following two papers stood out at the conference as high-impact applications.

"Smell Pittsburgh: Engaging Community Citizen Science for Air Quality" proposes a system for citizens to report pollution odors to the local health department in real time via smartphones. The system visualizes odor complaints so that residents can confirm their experiences with others and provides a comprehensive overview of the local pollution landscape. The system can even predict upcoming smell events and send push notifications to inform residents.

"Photo Sleuth: Identifying Historical Portraits with Face Recognition and Crowdsourced Human Expertise" presents an innovative web-based tool to support Civil War portrait identification. Although many thousands of American Civil War soldier portraits survived, only $10 \%$ to $20 \%$ of them were identified. By combining crowdsourced human expertise and automated face recognition, Photo Sleuth provides a sustainable model for volunteer contribution.

Last but not least, "Affect-Aware Word Clouds" studies the connection between font choices and color palettes in word clouds, and finds that both are powerful tools contributing to the affect associated with a word cloud. Building on this result, the authors present an affect-aware font and color palette selection method to help users make more informed choices.

Overall, this set of articles highlights the excellence of ACM IUI 2019 and the outstanding work and breadth of the community at the intersection of artificial intelligence and human-computer interaction. For readers who are new to this field, it is our hope that you find this special issue a launching point to your further exploration of intelligent human-machine interactions. For our seasoned researchers, we believe that the included papers make significant contributions to the scientific community to enable researchers, practitioners, and students working in the areas to further advance the theories and practices in this area.

We would like to extend special thanks and acknowledge the hard work of our authors, as well as the program committee and special issue reviewers, who thoroughly reviewed each paper and provided valuable feedback. We would also like to express our gratitude to Michelle Zhou, the editor-in-chief of ACM TiiS, and Liang Gou, the assistant to the editor-in-chief, for keeping us on track in putting together this special issue highlighting the best of ACM IUI 2019.

Oliver Brdiczka

Duen Horng Chau

Minsuk Kahng

Gaëlle Calvary

Guest Editors 